\title{
Le plan de vaccination suisse 2014
}

Office fédéral de la santé publique (OFSP) et Commission fédérale pour les vaccinations $(\mathrm{CFV})$
Le plan de vaccination suisse 2014 (Directives et recommandations) vient d'être publié (le 17 février 2014) [1]; voir Factsheet «Plan de vaccination suisse» en annexe. Sont présentées ici les seules nouveautés du plan de vaccination 2014, concernant la vaccination contre les pneumocoques, ainsi que les nombreuses adaptations de la vaccination contre la coqueluche depuis 2012.

\section{Nouveautés concernant la vaccination contre les pneumocoques à partir de 2014 [2]}

\section{Vaccination de base}

La vaccination généralisée contre les pneumocoques des personnes âgées de 65 ans et plus avec le vaccin polysaccharidique 23-valent (PPV23) n'est actuellement plus recommandée. La vaccination avec le PPV23 n'apporte d'une part que peu de bénéfices aux personnes sans facteur de risque de maladie invasive à pneumocoques. D'autre part, en cas d'apparition ultérieure de facteurs de risque, elle diminue la qualité de la réponse immunitaire induite par un vaccin conjugué 13-valent (PCV13) éventuellement indiqué (hyporéponse).

\section{Personnes à risque élevé de maladie invasive à pneumocoques (MIP)}

Après une analyse détaillée des données actuelles concernant l'épidémiologie des MIP en Suisse et l'immunogénicité des vaccins contre les pneumocoques, l'OFSP et la CFV ont défini les groupes à risque de manière plus précise (afin de cibler ceux qui sont le plus à risque, au meilleur moment) et recommandent la vaccination des personnes à risque élevé de MIP de la façon suivante:

- Une dose unique de vaccin conjugué 13-valent contre les pneumocoques PCV13 ( $>1$ dose chez les enfants de $<2$ ans et les receveurs d'une transplantation).

- Cette vaccination est recommandée avant la période de risque maximal, définie précisément pour chaque groupe à risque (pour les détails, voir [2] ou Factsheet «Groupes à risques» en annexe).

- Les rappels et vaccinations supplémentaires avec le vaccin polysaccharidique 23 -valent contre les pneumocoques (PPV23) ne sont actuellement pas nécessaires et donc plus recommandés.

Correspondance: Office fédéral de la santé publique OFSP Division Maladies transmissibles

Schwarztorstrasse 96

CH-3003 Berne Tél. 0313238706 patients immunosupprimés. Les précautions générales s'appliquent en cas de réaction allergique grave à une vaccination antérieure ou lors de maladie aiguë ou fébrile. Afin d'optimiser les réponses au PCV13, deux mesures sont recommandées:

- Respecter un intervalle minimum de 12 mois après la dernière dose de vaccin PPV23.

- Par mesure de précaution, respecter si possible un intervalle de 4 semaines avec un vaccin antigrippal.

\section{Remboursement de la vaccination}

La vaccination contre les pneumocoques avec le PCV13 n'est à la charge de l'assurance obligatoire des soins que pour les groupes d'âge pour lesquels le vaccin a reçu une autorisation de mise sur le marché de la part de Swissmedic. Dans l'attente d'une extension de l'autorisation du vaccin PCV13 au-delà de 5 ans, la vaccination PCV13 n'est donc pas à la charge de l'assurance obligatoire des soins après l'âge de 5 ans. L'information professionnelle prévoit cependant que «Prevenar $13^{\circledR}$ doit être utilisé selon les recommandations officielles qui tiennent compte de l'impact des infections invasives dans les différentes classes d'âge».

\section{Adaptations de la vaccination contre la coqueluche depuis 2012 [3, 4]}

Consignes générales: la vaccination contre la coqueluche $\left(\mathrm{P}_{\mathrm{a}}\right)$ chez les nourrissons et les enfants jusqu'à l'âge de 8 ans est effectuée avec des vaccins combinés en dosage pédiatrique $\left(\mathrm{DTP}_{\mathrm{a}}\right)$. Les enfants de 8 ans et plus et les adultes sont vaccinés avec un dosage plus faible pour les composantes coqueluche $\left(\mathrm{p}_{\mathrm{a}}\right)$ et diphtérie $(\mathrm{d})$ (vaccins combinés $\mathrm{dTp}_{\mathrm{a}}$, par ex. Boostrix $^{\circledast}$ ). On ne dispose d'aucun vaccin anticoquelucheux monovalent. En raison de l'évolution de la situation épidémiologique au cours des dernières années (augmentation de l'incidence principalement chez les enfants plus âgés, les adolescents et les adultes) et de la durée limitée de la protection immunitaire après la dernière vaccination ou infection, les nouvelles recommandations vaccinales suivantes ont été formulées depuis 2012 avec comme but premier de réduire le risque de transmission et de maladie chez les nourrissons.

\section{Vaccination de base}

Nourrissons: depuis 2013, un schéma accéléré à l'âge de 2 , 3 et 4 mois est recommandé pour les nourrissons qui iront dans une structure d'accueil collectif avant l'âge de 5 mois [3]. Après un schéma de vaccination accéléré, le premier rappel ( $4^{\mathrm{e}}$ dose) est indiqué dès l'âge de 12-15 mois (plutôt qu'à 15-24 mois). Ce schéma accéléré peut éventuellement être envisagé chez tous 
les nourrissons lors d'épidémies (voir également [5]).

Adolescents: depuis 2013, il est recommandé que les adolescents âgés de 11 à 15 ans bénéficient d'un rappel supplémentaire ( $6^{\mathrm{e}}$ dose) ou d'une primovaccination [3].

Pour éviter une hyperimmunisation, une vaccination contre la coqueluche avec un vaccin $d \mathrm{dp}_{\mathrm{a}}$ est recommandée chez les personnes de 11 à 15 ans déjà complètement vaccinées contre la diphtérie et le tétanos (y compris la dose due pendant l'adolescence) seulement si les conditions suivantes sont réunies:

1) moins de cinq doses anticoquelucheuses et

2) aucune vaccination contre la coqueluche depuis l'âge de 8 ans et

3) aucune vaccination dT au cours des deux dernières années.

Adultes: depuis 2012, une vaccination unique contre la coqueluche sous forme d'un rappel supplémentaire (7e dose) ou d'une primovaccination est recommandée chez les adultes de 25 à 29 ans (jusqu'au $30^{\mathrm{e}}$ anniversaire) [4]. L'intervalle minimal à respecter après la dernière dose contre le tétanos est de deux ans.

Si une prophylaxie antitétanique est indiquée en cas de blessure chez les adolescents et les adultes de 25 à 29 ans, l'opportunité doit être saisie pour administrer un vaccin combiné avec un composant coqueluche $\left(\mathrm{dTp}_{\mathrm{a}}\right)$.

\section{Vaccinations recommandées dans les situations à} risque / chez les personnes à risque

Adolescents et adultes: une vaccination immédiate contre la coqueluche est recommandée indépendamment de l'âge chez les adolescents et les adultes en cas de contacts réguliers (au travail / dans la famille) actuellement ou dans un futur proche avec des nourrissons de moins de 6 mois et si la dernière vaccination contre cette maladie ou infection confirmée par laboratoire remonte à dix ans ou plus [4]. L'intervalle minimal après la dernière vaccination antitétanique est alors de quatre semaines.

Depuis 2013, une vaccination contre la coqueluche est recommandée chez les femmes enceintes au cours du deuxième ou troisième trimestre si la dernière vaccination contre cette maladie ou infection confirmée par laboratoire remonte à plus de cinq ans [3]. En cas d'absence de vaccination avant ou pendant la grossesse, les femmes devraient être vaccinées contre la coqueluche aussi rapidement que possible après l'accouchement. L'intervalle minimal après la dernière vaccination antitétanique est de quatre semaines.

\section{Remboursement de la vaccination}

La vaccination de base contre la coqueluche et la vaccination contre la coqueluche chez les personnes à risque / dans les situations à risque sont remboursées par l'assurance obligatoire des soins, ou par l'assurance-accident lorsqu'une vaccination post-expositionnelle contre le tétanos est indiquée après un accident et qu'une vaccination concomitante contre la coqueluche est recommandée selon le plan vaccinal. Lorsque la vaccination est indiquée en raison des activités professionnelles exercées, le coût est en général pris en charge par l'employeur.

\section{Information supplémentaire}

En annexe de ce numéro: la fiche d'information «Plan de vaccination suisse» (état février 2014) et «Groupe à risque». Ces deux documents sont disponible en trois langues: français, allemand et italien. Le plan de vaccination suisse 2014 (version complète) et les factsheets peuvent être téléchargés sur les sites Internet de l'OFSP (www.sevacciner.ch $\rightarrow$ Informations et matériel pour les professionnels de santé) ou d'InfoVac (www.infovac.ch).

\section{Références}

1 Office fédéral de la santé publique, Commission fédérale pour les vaccinations. Plan de vaccination suisse. Directives et recommandations. Berne; 2014.

2 Office fédéral de la santé publique, Commission fédérale pour les vaccinations. Vaccination contre les pneumocoques: recommandations visant à prévenir les maladies invasives à pneumocoques chez les groupes à risque. Bull OFSP. 2014;8:129-41.

3 Office fédéral de la santé publique, Commission fédérale pour les vaccinations. Optimisation des rappels vaccinaux contre la diphtérie, le tétanos et la coqueluche (dT/dTpa) chez l'adulte. Bull OFSP. 2011;51:1161-71.

4 Office fédéral de la santé publique, Commission fédérale pour les vaccinations. Adaptation des recommandations de vaccination contre la coqueluche: pour les adolescents, les nourrissons fréquentant une structure d'accueil collectif et les femmes enceintes. Bull OFSP. 2013;9:118-23.

5 Office fédéral de la santé publique. Mesures de prévention et de contrôle des flambées de coqueluche dans les établissements de santé et les structures d'accueil collectif pour la protection des nourrissons de moins de 6 mois. Bull OFSP. 2013;13:188-192. 